\title{
Eficacia del coadyuvante orgánico Ecotensor SYS, en el control de Sigatoka negra (Mycosphaerella fijiensis) en cultivo de plátano (Musa AAB simmonds)
}

Efficacy of the organic Ecotensor SYS adjuvant, in the control of black Sigatoka (Mycosphaerella fijiensis) in plantain cultivation (Musa AAB simmonds)

\section{Eficácia do Ecotensor SYS orgânico adjuvante, no controle da Sigatoka} negra (Mycosphaerella fijiensis) no cultivo de banana (Musa AAB simmonds)

Casas Ovalle Danna Lorena ${ }^{1}$, Hernández Guevara Bayardo Enrique ${ }^{1}$, Pachón González Enrique William² y Martínez Martínez Edgar Alejo ${ }^{2}$

${ }^{1}$ Ingenieras Agrónomas, Universidad de los Llanos y

${ }^{2}$ Ingenieros Agrónomos Docentes Universidad de los Llanos

\section{danna.casas.ovalle@unillanos.edu.co}

Recibido 10 de febrero 2021, Aceptado 23 de abril 2021

\section{RESUMEN}

Se realizó un estudio cuyo propósito fue determinar la eficacia del coadyuvante orgánico (Ecotensor SYS) en mezcla con un fungicida de uso agrícola, estableciendo dosis para recomendación y evaluando posibles efectos de fitotoxicidad de Ecotensor en plantas de plátano (Musa AAB Simmonds); ensayo que se realiza para el control de Mycosphaerella fijiensis en el cultivo de plátano (Musa AAB Simmonds). Las pruebas se efectuaron en campo abierto en parcelas experimentales del cultivo, en el departamento de Meta, en los municipios de Granada y Fuente de Oro, cada parcela tuvo un área de $250 \mathrm{~m}^{2}$ con un promedio de 10 metros de ancho por 25 metros de largo, el área total fue $1000 \mathrm{~m}^{2}$, para cada ensayo. Se realizó un diseño experimental completamente al azar. Los resultados evidencian que las aplicaciones de fungicida con Ecotensor, proporcionan un mejor nivel de control de la enfermedad en comparación con los tratamientos de fungicida con Hipotensor y fungicida sin coadyuvante. La aplicación de Ecotensor SYS no generó daños ni síntomas de fitotoxicidad sobre el follaje de las plantas de plátano (Musa $A A B$ Simmonds). Por lo tanto, se concluye que se obtienen ventajas 
favorables al utilizar fungicida acompañado del coadyuvante orgánico Ecotensor evaluado, y que la dosis para recomendación de aplicación es de $1.0 \mathrm{ml} / \mathrm{l}$ de agua.

Palabras clave: Fitotoxicidad, fungicida, enfermedad, grado de severidad.

\begin{abstract}
A study was carried out whose purpose was to determine the efficacy of the organic adjuvant (Ecotensor SYS) in mixture with a fungicide for agricultural use, establishing doses for recommendation and evaluating possible effects of phytotoxicity of Ecotensor in banana plants (Musa $A A B$ Simmonds); test carried out for the control of Mycosphaerella fijiensis in plantain cultivation (Musa $A A B$ Simmonds). The tests were carried out in open field in experimental plots of the crop, in the department of Meta, in the municipalities of Granada and Fuente de Oro, each plot had an area of $250 \mathrm{~m}^{2}$ with an average of 10 meters wide by 25 meters long, the total area was $1000 \mathrm{~m}^{2}$, for each trial. A completely randomized experimental design was carried out. The results show that the fungicide applications with Ecotensor provide a better level of disease control compared to the fungicide treatments with hypotensor and fungicide without adjuvant. The application of SYS Ecotensor did not generate damage or symptoms of phytotoxicity on the foliage of banana plants (Musa AAB Simmonds). Therefore, it is concluded that favorable advantages are obtained when using fungicide accompanied by the evaluated organic adjuvant Ecotensor, and that the dose for application recommendation is 1,0 $\mathrm{ml} / \mathrm{l}$ of wáter.
\end{abstract}

Keywords: Phytotoxicity, fungicide, disease, degree of severity.

\title{
RESUMO
}

Foi realizado um estudo cujo objetivo foi determinar a eficácia do adjuvante orgânico (Ecotensor SYS) em mistura com um fungicida para uso agrícola, estabelecendo doses para recomendação e avaliando possíveis efeitos de fitotoxicidade do Ecotensor em bananeira (Musa AAB Simmonds); teste realizado para o controle de Mycosphaerella fijiensis no cultivo de banana (Musa $A A B$ Simmonds). Os testes 
foram realizados em campo aberto em parcelas experimentais da cultura, no departamento de Meta, nos concelhos de Granada e Fuente de Oro, cada parcela tinha uma área de $250 \mathrm{~m}^{2}$ com uma média de 10 metros de largura por 25 metros de comprimento., a área total era de $1000 \mathrm{~m}^{2}$, para cada ensaio. Foi realizado um delineamento experimental inteiramente casualizado. Os resultados mostram que as aplicações de fungicida com Ecotensor proporcionam um melhor nível de controle da doença em comparação aos tratamentos com fungicida com hipotensor e fungicida sem adjuvante. A aplicação do Ecotensor SYS não gerou danos ou sintomas de fitotoxicidade na folhagem da bananeira (Musa AAB Simmonds). Portanto, conclui-se que vantagens favoráveis são obtidas ao se utilizar fungicida acompanhado do adjuvante orgânico Ecotensor avaliado, e que a dose para recomendação de aplicação é de $1.0 \mathrm{ml} / \mathrm{l}$ de água.

Palavras-chave: Fitotoxicidade, fungicida, doença, grau de severidade.

\section{INTRODUCCIÓN}

producción de plátano (Musa AAB Simmonds) y banano (Musa spp) representa una de las actividades socio económico más importante de los países de América Latina El cultivo de plátano (Musa spp) en Colombia representa cerca del $50 \%$ del área sembrada en el país con cerca de 500 mil hectáreas cultivadas, y aunque es un fruto que se da en todo el territorio colombiano, su producción es principalmente para el consumo interno (Espinal et al., 2005).

Este cultivo se ve afectado por una enfermedad de importancia económica la "Sigatoka Negra" causada por el hongo Mycosphaerella fijensis, la cual está considerada como uno de los problemas fitopatológicos más importantes que afectan la producción y su control incide fuertemente en los costos de producción. (Rivas y Rosales, 2003).

En plantaciones con bajo nivel de infección, los síntomas de "Sigatoka Negra" pueden ser fácilmente confundidos con los síntomas de Sigatoka Amarilla, especialmente en plantas jóvenes o en colinos bandera, donde las manchas individuales presentan una apariencia circular a ovalada de igual color y apariencia. 
En ataques severos, la "Sigatoka Negra" es inconfundible en platas desarrolladas aun sin racimo, por la gran cantidad de rayas y manchas de color café a negro que pueden cubrir toda el área foliar en forma descendente desde la tercera hoja más joven abierta (González et al., 2012).

El ciclo de vida de $M$. fijensis se inicia con la deposición de las ascosporas o conidios del hongo, que ha sido liberadas por el viento, sobre las hojas libres de la enfermedad. Bajo condiciones favorables de humedad, temperatura y en presencia de agua libre en la superficie de la hoja, el proceso de germinación ocurre en una hora aproximadamente. La penetración al hospedero está condicionada por el tiempo que dure la película de agua sobre la hoja y la humedad relativa, pero normalmente ocurre en un lapso de dos a tres días. El período de incubación del hongo, referido como el tiempo entre germinación y aparición de la primera pizca dura en banano es de 17 días y en plátano 29 días, mientras que el período de latencia o sea hasta la aparición de conidióforos y conidios, que se forman en el estado de estría, ocurre 28 días luego de la infección en banano y 34 días después en plátano. Las ascosporas maduras de M. Fijensis se pueden observar 49 días después de la infección en banano y 64 días después en plátano. Durante los meses de verano los períodos de incubación y latencia, la transición infección a cada uno de los síntomas y la formación de peritecios presentan una mayor duración promedio, que se refleja en un retardo en la manifestación de síntomas y por ende en la formación de conidios, peritecios y ascosporas (Orozco et al., 2008).

Las condiciones ambientales, el estado fisiológico y grado de nutrición de la plata, la virulencia del patógeno junto con la concentración de esporas o conidias, son determinantes en la intensidad de la infección y la evolución a cada uno de los estadios de desarrollo de la enfermedad (Cuéllar et al.,2011).

Sobre las manchas que caracterizan esta enfermedad se producen dos tipos diferentes de inóculo que corresponden al estado asexual y sexual del patógeno, siendo en el estado conidias, empieza a formarse en el campo desde el estado de estría hasta formar mancha. Conteos de conidióforos y conidias en cada estado de 
desarrollo de la enfermedad, indican que una mayor cantidad de conidióforos se producen en el segundo estado de estría (Orzco et al., 2013).

Sobre este tipo de lesiones no fue posible contabilizar la cantidad de conidios formados, debido a que son fácilmente liberados por el viento y el agua; sin embargo, en plátano, sobre una estría estado dos, con un área entre 7 y $30 \mathrm{~mm}^{2}$ pueden existir cerca de siete estomas por milímetro cuadrado, de los cuales un $75 \%$ aparecen formando conidióforos con cerca de cinco cosechas de conidios. Formaciones de sólo un conidóforo en el $50 \%$ de las estomas, sugieren que la habilidad esporulativa del patógeno podría llegar a generar entre 100 y 300 conidios (Orzco et al., 2013).

Las infecciones son más importantes en el envés de la hoja debido a que los estomas son más numerosos en esta parte de la hoja y cuando la hoja se desenvuelve, su envés es la parte que se expone primero a los propágulos del hongo. Luego viene el período de incubación, el cual se encuentra muy estrechamente relacionado con las condiciones climáticas y posteriormente el desarrollo visible de la enfermedad, la cual abarca desde el primer estadio hasta la necrosis. Las manchas comienzan a secarse, originando depresión en el tejido enfermo; es común que a partir de este estado las manchas presenten amarillamiento del tejido circundante.

La mancha original se seca completamente y adquiere un color café claro, que con el tiempo llega a tonalidades aún más claras. La m1ancha en sí, se rodea de un borde oscuro y tejido clorótico. En casos severos de infección, las hojas enfermas se secan y mueren dentro de las tres a cuatro semanas siguientes a la aparición de los primeros síntomas. En tales casos las plantas antes de la cosecha llegan a perder la totalidad de su follaje (Álvarez et al., 2013).

Un coadyuvante es cualquier sustancia agregada al tanque de la pulverizadora, en forma separada de la formulación del pesticida, a fin de mejorar el rendimiento del mismo (Sazo et al., 2008). El uso de coadyuvantes ha sido dedicado solo a la mezcla con herbicidas postemergentes, sin embargo, muchos otros agroquímicos como 
fungicidas, insecticidas, reguladores de crecimiento y abonos foliares, pueden mejorar su efecto cuando se le adicionan coadyuvantes a la mezcla de tanque (Arrospide, 2004).

Los coadyuvantes ayudan a mejorar la cobertura (mojado) y la persistencia (pegajosidad) del ingrediente activo, o de los elementos minerales en la superficie de las hojas, así también como promover mayor velocidad de absorción y bioactividad de los elementos minerales aplicados. Las limitaciones a la absorción foliar de los elementos minerales aplicados han conducido a un amplio uso y búsqueda continua de coadyuvantes que mejoren la efectividad de los tratamientos de pulverización foliar (Fernández et al., 2015).

En el mercado existen muchos coadyuvantes de diferentes compuestos y con diferentes modos de acción para realizar funciones específicas, incluyendo ajuste del $\mathrm{pH}$, dispersión, extensión, pegado y humectación. Estos compuestos también pueden reducir la evaporación, la formación de espuma, la deriva de la pulverización y la volatilización, pero ningún coadyuvante solo puede realizar todas estas funciones, pero a menudo pueden combinarse para realizar múltiples funciones al mismo tiempo, Por lo tanto, su uso no sólo ayuda a minimizar problemas de las aplicaciones, sino también aumentar la eficiencia de los mismos. Debido a que se consideran como sustancias inertes en las formulaciones químicas, no existe una regulación para los coadyuvantes (INTAGRI, 2017).

Por lo general, los coadyuvantes se clasifican como: activadores y utilitarios, donde los primeros aumentan la actividad, penetración, difusión y retención del ingrediente activo, mientras que los utilitarios solo modifican la propiedad de la solución sin afectar directamente la eficiencia de la formulación. Aunque sus efectos son diversos, todos estos materiales contienen moléculas que tienen grupos polares como no polares. En soluciones de aceite o agua, estas moléculas pueden alinearse con el grupo apolar en el aceite y el grupo polar en el agua. Debido a esta propiedad los coadyuvantes tienen varios efectos: 1- Proporcionan una mayor área de contacto de cada gota de agua, y se aumenta la absorción del producto químico.2- Aumentan la penetración de agroquímicos en la cutícula, abriendo espacios intermoleculares, 
o haciendo a los agroquímicos polares y las moléculas acuosas más solubles en las ceras apolares de la cutícula (Ramos y Peñaranda, 2018).

El Ecotensor SYS es un coadyuvante agrícola líquido, $\mathrm{pH}, 3.0-3.5$ ligeramente viscoso a $25^{\circ} \mathrm{C} 77^{\circ} \mathrm{F}$ cP 290 , amarillo, Densidad a $25^{\circ} \mathrm{C}\left(77^{\circ} \mathrm{F}\right), 1.031 \mathrm{~g} / \mathrm{ml}$. Siendo su fórmula su química y concentración L-glutamic acid, N-coco acyl derivates 100 $\mathrm{g} / \mathrm{l}$ agua, con un surfactante no iónico. Dispersable en agua, ligeramente soluble en hidrocarburos alifáticos, miscible en la mayoría de solventes polares orgánicos e hidrocarburos aromáticos, químicamente estable en la mayoría de soluciones alcalinas y acidas, compatible con surfactantes aniónicos, catiónicos y otros surfactantes no iónicos.

Con relación a lo anterior el objetivo de esta investigación fue determinar la eficacia del coadyuvante orgánico (Ecotensor SYS) en mezcla con un funguicida a base de Fenpropidin $750 \mathrm{~g} / \mathrm{l}$ de uso agrícola, con el fin de establecer su grado emulsionante, penetrante y surfactante para el tratamiento de Sigatoka negra (Mycosphaerella fijiensis Morelet) en el cultivo de plátano.

\section{METODOLOGÍA}

\section{Ubicación}

El trabajo experimental se realizó en el municipio de Granada y en el municipio de Fuente de Oro en el departamento del Meta, Colombia - Suramérica. En esta zona los veranos son cortos, muy caliente, húmedo y nublados; los inviernos son cortos, caliente y parcialmente nublados y está mojado durante todo el año. Durante el transcurso del año, la temperatura generalmente varía de 19 a $31^{\circ} \mathrm{C}$ y rara vez baja a menos de $15^{\circ} \mathrm{C}$ o sube a más de $34^{\circ} \mathrm{C}$.

En esta zona los suelos son aptos para el desarrollo del cultivo del plátano (Musa $A A B$ Simmonds), siendo su textura franco arenosa, franco arcillosa, franco arcillo limosa y franco limoso, además, fértiles, permeables, profundos (1.2-1.5 $\mathrm{m}$ ), bien drenados y ricos especialmente en materias nitrogenadas. Es de anotar que este cultivo tiene una gran tolerancia a la acidez del suelo, oscilando el pH entre 4.5-8, 
siendo el óptimo 6.5. Por otra parte, los plátanos se desarrollan mejor en suelos planos, como son los de esta zona (Mejia, 2018).

\section{Tratamientos en parcelas experimentales:}

Se seleccionaron las parcelas y los tratamientos se establecieron completamente al azar; cada parcela tuvo un área de $250 \mathrm{~m}^{2}$ con un promedio de 10 metros de ancho por 25 metros de largo, El área total fue $1.000 \mathrm{~m}^{2}$, para cada ensayo. Las pruebas se efectuaron en campo abierto en parcelas experimentales de cultivo de plátano, en época de invierno en el Departamento de Meta. Las pruebas se realizaron en dos fincas ubicadas en Granada y Fuente de oro que son representativas del cultivo y con alta incidencia de la enfermedad de la Sigatoka negra (Mycosphaerella fijiensis Morelet).

Las plantas estudiadas fueron las sometidas a infección natural de la enfermedad, y las localidades, y no se aplicaron otros fungicidas durante su desarrollo. La variable de respuesta fue el grado de severidad medido cada cinco días, después de la primera aplicación. En la primera evaluación de los resultados se analizó el crecimiento de la Sigatoka en las plantas seleccionadas, se realizó un seguimiento a cada planta por individual. En la segunda evaluación se detalló el estado final de las plantas seleccionadas para esta investigación.

Los tratamientos se especifican en la Tabla 1. Se utilizaron diferentes dosis del coadyuvante orgánico (Ecotensor SYS) que es un líquido viscoso, de color amarillo $\mathrm{pH}, 3.0-3.5$, densidad a $25^{\circ} \mathrm{C}\left(77^{\circ} \mathrm{F}\right), 1.031 \mathrm{~g} / \mathrm{ml}$. Siendo su fórmula su química y concentración L-glutamic acid, N-coco acyl derivates y su $100 \mathrm{~g} / \mathrm{l}$ agua, con un surfactante no iónico, este se mezcló en mezcla con un funguicida a base de Fenpropidin de uso agrícola, utilizando $600 \mathrm{ml}$ por hectárea, además se utilizó un tratamiento con un coayudante de referencia el Hipotensor SYS, ya está probado que $1 \mathrm{ml} / /$ de agua es efectivo para el tratamiento de la sigatoka negra cuando se utiliza en mezcla con el fungicida; este producto está compuesto a base de Polyoxyethylene (6) Lineal alcohol (9-11) $100 \mathrm{~g} / \mathrm{l}$, de tipo de surfactante, no iónico, liquido ligeramente viscoso de color amarillo y con un $\mathrm{pH}$, 3.0-3.5. (Ramos y 
Peñaranda, 2018). El modo de aplicación fue mediante aspersión foliar, con bomba manual de espalda, de presión constante. Después de realizada la aplicación se realizaron dos evaluaciones a los cinco y 10 días después de la aplicación.

Tabla 1. Tratamientos experimentales en Granada y Fuente de Oro

\begin{tabular}{ccc}
\hline Tratamiento & Coadyuvante & Dosis $\mathbf{~ m I} / \mathbf{l}$ \\
\hline Control & Fungicida únicamente & \\
1 & Hipotensor SYS & 1,0 \\
2 & Ecotensor SYS & 0,5 \\
3 & Ecotensor SYS & 1,0 \\
4 & Ecotensor SYS & 1,5 \\
\hline
\end{tabular}

El coayudante Ecotensor e hipotensor se mezclaron con el fungicida $600 \mathrm{cc} / \mathrm{ha}$

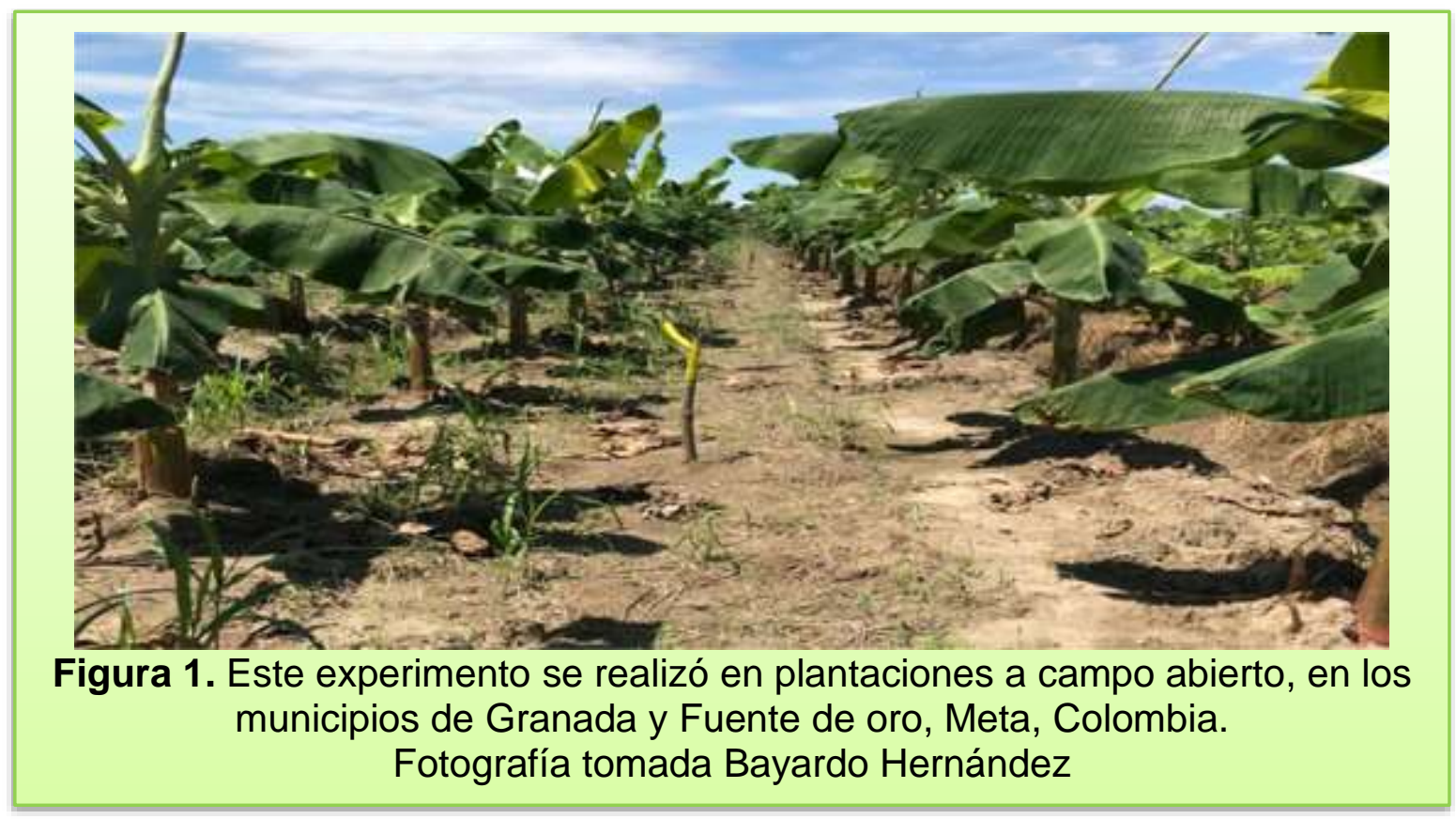

\section{Evaluación visual de la severidad de la fitotoxicidad}

Las plantas al inicio del ensayo tuvieron un mínimo de cuatro hojas funcionales garantizando un normal desarrollo agronómico del cultivo, con aplicaciones manuales dirigidas a todas las hojas de las plantas. Se evaluaron visualmente los folíolos de 10 hojas en cada tratamiento y se calculó el porcentaje de cada 
replicación y para determinar la severidad se utilizaron los diagramas estándares de Stover, (1971) y la escala de grados de desarrollo de las Sigatokas (Aranzazu et al., 2002), lo cual se realizó en las hojas de las plantas en las cuales se les hacia el seguimiento (Tabla 2).

Tabla 2. Grados y síntomas de fitotoxicidad

\begin{tabular}{cl}
\hline Puntuación & \multicolumn{1}{c}{ Síntoma de Fitotoxicidad } \\
\hline 1 & Ausencia de Síntomas (Planta Sana). \\
2 & síntomas muy leves, cierta atrofia, amarillamientos, etc. \\
3 & Síntomas leves, cierta atrofia, amarillamientos claramente apreciables. \\
4 & Clorosis y/o atrofia más aguda. Sin influir en el desarrollo del cultivo. \\
5 & $\begin{array}{l}\text { Reducción de la población, fuerte clorosis y/o atrofia, se observa } \\
\text { Influencia en el desarrollo. }\end{array}$ \\
6 & Daños crecientes hasta la detención del crecimiento del cultivo \\
7 & Daños crecientes hasta la muerte parcial del cultivo. \\
8 & Daños crecientes hasta la muerte total del cultivo. \\
9 & Muerte total del cultivo. \\
\hline
\end{tabular}

Se realizó la evaluación de fitotoxicidad en parcelas independientes que tenían la misma variedad utilizada en la prueba de eficacia, sobre la cual se realizó una única aplicación en 160 plantas en diferentes dosis del producto a evaluado. En total se utilizaron 80 plantas por localidad.

El porcentaje de severidad se obtuvo según la escala de severidad de Stover que consiste en evaluar el porcentaje de afección en las hojas seleccionadas, los rangos se especifican en la Tabla 3.

\section{Análisis Estadístico}

Se utilizó un diseño de cuatro bloques completos con cuatro tratamientos, para conformar 16 unidades experimentales, cada una tenía cuatro plantas para un total de 64 plantas por cada finca (Granada y Fuente de Oro) y cinco repeticiones; para el estudio del análisis de varianza de un factor (Evaluación de la fitotoxicidad) de medias repetidas, cumpliendo la revisión de los postulados movilidad, normalidad, 
homogeneidad de varianza y esfericidad (Prueba de Mauchly), se elaboraron gráficas de los resultados, también se hizo análisis de la prueba de comparación de Duncan.

Tabla 3. Porcentaje de severidad según Stover

\begin{tabular}{ccc}
\hline $\begin{array}{c}\text { Grado de } \\
\text { severidad }\end{array}$ & $\begin{array}{c}\text { Porcentaje de afección en } \\
\text { la hoja }\end{array}$ & Estado de la enfermedad \\
\hline 0 & 0 & Hojas sin síntomas \\
1 & Menos de 50 Pizcas & Pizca \\
2 & $1-5$ & \\
3 & $6-15$ & Área foliar afectada \\
4 & $16-33$ & \\
5 & $34-50$ & \\
6 & Mayor de 50 & \\
\hline
\end{tabular}

\section{RESULTADOS Y DISCUSIÓN}

Los daños causados por la sigatoka negra en las hojas de plátano se evaluaron realizando unas series de dibujo de acuerdo a la sintomatología que se detalla en la Tabla 2, se midieron las manchas y de esta forma se calculó el porcentaje de destrucción de hojas. La intensidad de la afección se clasifico en cinco grados, tal como se especificó en la Tabla 3, esta metodología con la cual Stover, (1971) desarrolló una escala que es utilizada a nivel internacional, no solo en plantaciones de banano, sino que también es aplicable para evaluar la severidad de esta enfermedad en cultivos de plátano, lo que hace posible comparar los resultados de los experimentos realizados en diferentes partes del mundo.

A los cero días antes de iniciar los tratamientos todas las plantas que se estudiaron tenían un bajo índice de afectación por la sigatoka negra, grado dos, porque ninguna planta fue superior a $11.9 \%$ de acuerdo a escala de medición de Stover, (1971) por lo tanto, estaban las condiciones uniformes para la aplicación de loa tratamientos y de esta manera medir su efecto de manera eficiente y real, puesto como se explicó el experimento fue realizado a campo abierto (Tabla 4 y Figuras 2 y 3 ). A los cinco 
días después de aplicados los tratamientos el control que únicamente tenía fungicida, presento una severidad mayor $(P<0.05)$ de afectación de la sigatoka negra de grado $4.24 \%$, para Granada y $18.8 \%$ para Fuente de oro, en comparación con los demás tratamientos, los cuales mostraron un comportamiento similar (Tabla 4 y Figura 4). Aunque no se observaron diferencias cuando se utilizaron Hipotensor SYS y Ecotensor SYS en varias dosis, es de anotar que se observan mayores porcentajes de afectación de la hoja en Granada en comparación con Fuente de oro (Tabla 4).

El cultivo del plátano a los 10 días el tratamiento control en Granada observó un grado cinco de afectación $(P<0.05) 34.5 \%$ (Figura 6 ) mientras que en Fuente de oro un grado cuatro, $19.5 \%$, siendo superior el daño de la sigatoka negra $(P<0.05)$ en comparación con los demás tratamientos, en los cuales presentaron un grado de severidad de tres (Tabla 4 y Figura 5).

Tabla 4. Índice de infección (\%) en la hoja de plátano por sigatoka negra (Mycosphaerella fijensis)

\begin{tabular}{ccccccc}
\hline \multirow{2}{*}{ Tratamientos } & \multicolumn{2}{c}{ Cero días } & \multicolumn{2}{c}{ Cinco días } & \multicolumn{2}{c}{ Diez días } \\
\cline { 2 - 7 } & Granada & $\begin{array}{c}\text { Fuente de } \\
\text { Oro }\end{array}$ & Granada & $\begin{array}{c}\text { Fuente de } \\
\text { Oro }\end{array}$ & Granada & $\begin{array}{c}\text { Fuente de } \\
\text { Oro }\end{array}$ \\
\hline Control & $7.2^{\mathrm{a}}$ & $11.9^{\mathrm{a}}$ & $24.5^{\mathrm{b}}$ & $18.8^{\mathrm{b}}$ & $34.5^{\mathrm{b}}$ & $19.5^{\mathrm{b}}$ \\
Hipotensor $1.0 \mathrm{ml}$ & $8.6^{\mathrm{a}}$ & $8.0^{\mathrm{a}}$ & $9.6^{\mathrm{a}}$ & $10.0^{\mathrm{a}}$ & $13.6^{\mathrm{a}}$ & $12.6^{\mathrm{a}}$ \\
Ecotensor $0.5 \mathrm{ml}$ & $9.6^{\mathrm{a}}$ & $8.3^{\mathrm{a}}$ & $12.2^{\mathrm{a}}$ & $10.4^{\mathrm{a}}$ & $13.6^{\mathrm{a}}$ & $14.1^{\mathrm{a}}$ \\
Ecotensor $1.0 \mathrm{ml}$ & $7.0^{\mathrm{a}}$ & $7.4^{\mathrm{a}}$ & $8.2^{\mathrm{a}}$ & $8.0^{\mathrm{a}}$ & $10.2^{\mathrm{a}}$ & $9.0^{\mathrm{a}}$ \\
Ecotensor $1.5 \mathrm{ml}$ & $11.0^{\mathrm{a}}$ & $9.2^{\mathrm{a}}$ & $13.1^{\mathrm{a}}$ & $10.8^{\mathrm{a}}$ & $15.6^{\mathrm{a}}$ & $14.4^{\mathrm{a}}$ \\
\hline
\end{tabular}

El coayudante Ecotensor e hipotensor se mezclaron con el fungicida $600 \mathrm{ml} / \mathrm{ha}$. El tratamiento control no contenía coayudante.

Se observa un incremento lineal en el porcentaje de afección de (Mycosphaerella fijensis) para el tratamiento control (600 $\mathrm{ml}$ de fungicida/ha), después de la segunda medición se generó resurgencia o reinfección a los 10 días, de igual forma sucedió en Granada y en Fuente de Oro (Tabla 1). 
El tratamiento con Ecotensor $1.0 \mathrm{ml}$ presenta el mejor control de Sigatoka, controlando el avance de la enfermedad en el tiempo de la evaluación, evidenciando los beneficios del uso del coadyuvante. En Ganada y Fuente de Oro del 7 y $7.4 \%$ de afección se incrementó levemente a 8.2 y $8.0 \%$ a los cinco días y $10.2 \%$ y $9 \%$ a los diez días de tratamiento, lo cual indica que con el coayudante Ecotensor SYS en concentraciones de $1.0 \mathrm{ml}$ en mezcla con el fungicida, se puede controlar esta enfermedad evitando una severidad y afectación mayor (Tabla 4 y Figura 5).

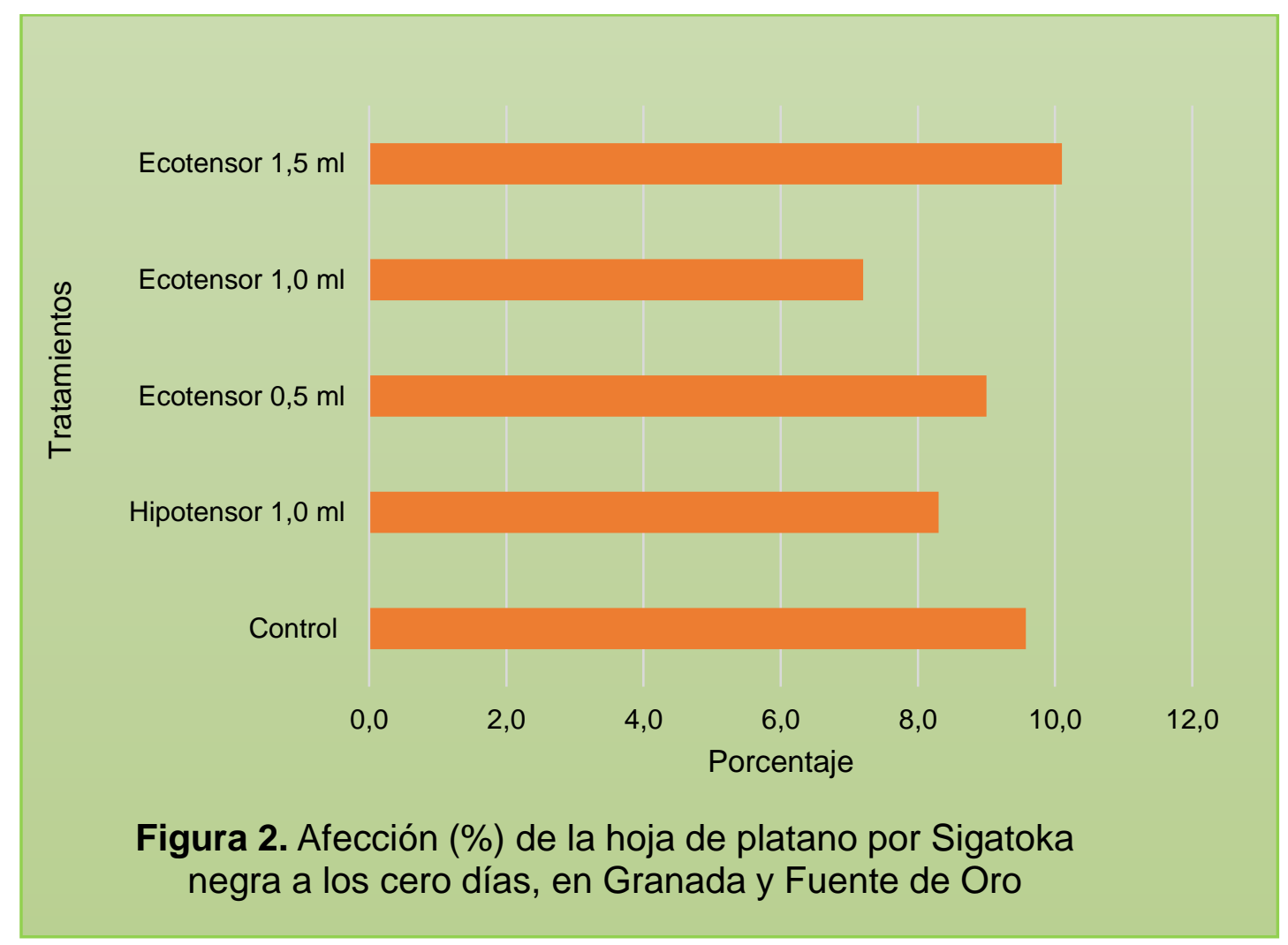

Esta zona donde se realizó este trabajo tiene las condiciones adecuadas para que la sigatoka negra sea progresiva si no se realiza un programa de control a su debido tiempo, es importante señalar que una mayor cantidad de hojas disponibles pueden permitir una infección más severa, puesto que las hojas verdaderas son emitidas aproximadamente a los 30 días, y el tiempo transcurrido desde la inoculación hasta la presentación de las manchas producidas por este hongo, es de 29 días (Álvarez et al., 2013). La sigatoka negra es una enfermedad altamente contagiosa, por lo tanto, su diseminación por las parcelas fue de pocos días como se observó en el 
tratamiento control a los 10 días de observación, principalmente en Granada, las altas humedades y temperaturas, facilitan la germinación de conidias y ascosporas (Figuras 5 y 6 ).

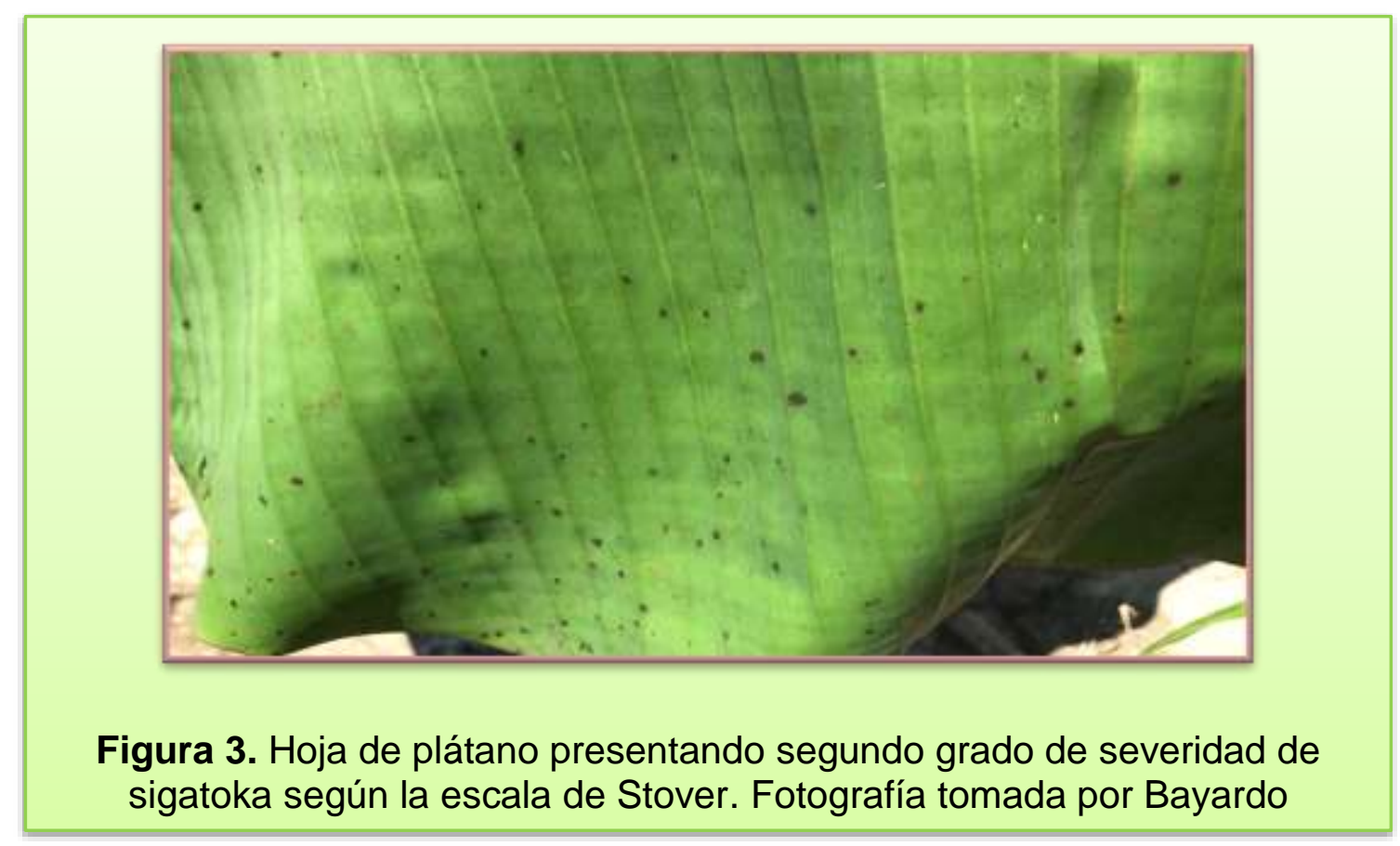

Las ascosporas y conidios requieren de temperaturas óptimas de entre 25 a $28^{\circ} \mathrm{C}$ para su máxima proliferación, mientras las ascosporas requieren de humedad relativa de 98 a 100\%, el ámbito para los conidios es más amplio de 92 a 100\% (Aguirre et al., 2012). El proceso de germinación en menos de dos horas y la penetración del tubo germinativo por la vía estomática se realiza en un periodo de una semana (Alarcón y Jiménez, 2012). Es por la situación explicada anteriormente que en menos de 10 días se observó un acelerado progreso de la Sigatoka negra, cuando no fue efectivo el tratamiento en este caso el control (Tabla 4, Figuras $5 \mathrm{y}$ 6 ), es de anotar que la viabilidad de los conidios sobre la superficie de la hoja puede ser mayor a los 60 días y que temperaturas superiores a $30^{\circ} \mathrm{C}$, pueden causar la desnaturalización de las proteínas presentes en la pared de las esporas del patógeno. 

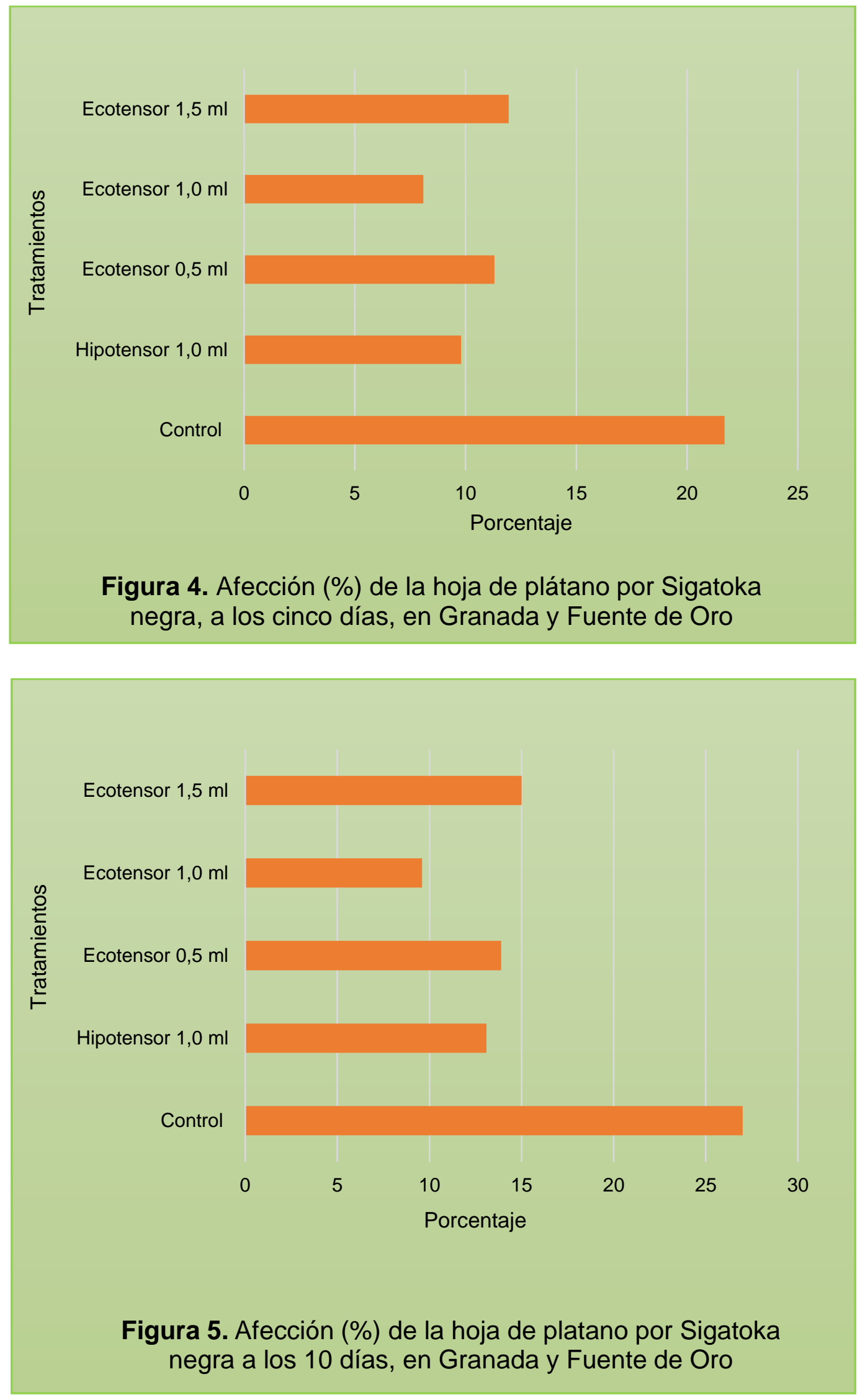


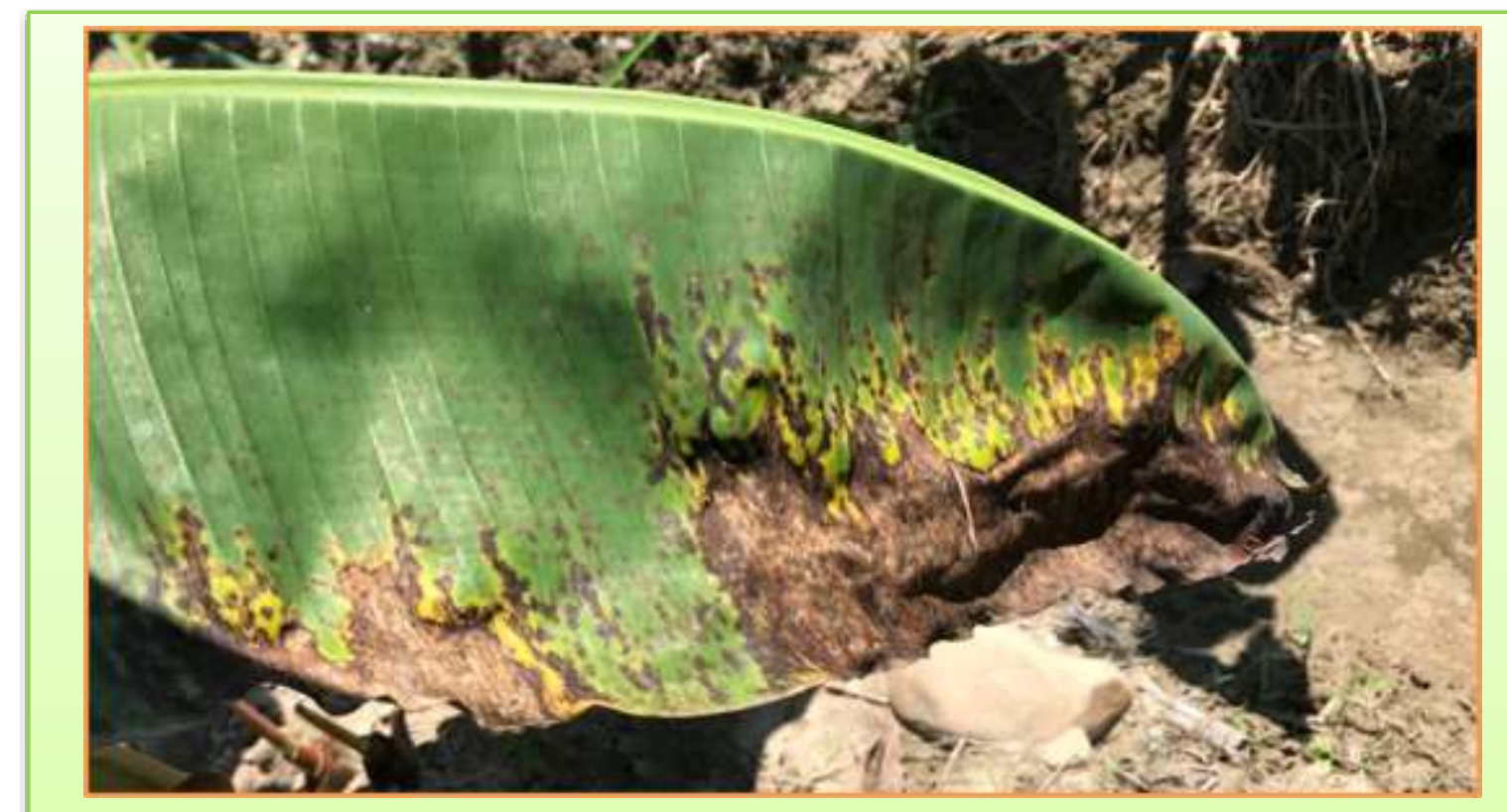

Figura 6. Hoja de plátano presentando quinto grado de severidad de sigatoka según la escala de Stover. Fotográfia tomada por Danna Casas.

De acuerdo a los resultados con el uso de los coayuvantes Hipotensor $(1.0 \mathrm{ml} / \mathrm{l}) \mathrm{y}$ Ecotensor en varias dosis, se observó que la Sigatoka negra no avanzara, es importante tener en cuenta que la presencia de partículas de arcilla, limo y materia orgánica suspendidas en el agua reducen la actividad en forma drástica de algunos productos de uso agrícola y además pueden estas partículas formar una película sobre la superficie foliar. El efecto de inactivación producido por las partículas en suspensión, no puede ser superado por el agregado de aditivos por lo que es importante seleccionar una fuente de agua que no presente problemas de esta índole. Es necesario aclarar que los coadyuvantes permiten engrosar la superficie de la hoja para evitar el escurrimiento lo que permite la acumulación de una mayor cantidad del producto motivo de la aplicación, lo cual puede redundar en mayor efectividad y residualidad del producto aplicado (Arrospide, 2004).

\section{CONCLUSIONES}

El cultivo del plátano (Musa AAB Simmonds) a los 10 días de experimentación el tratamiento, el control que contenía únicamente el fungicida, la Sigatoka negra 
(Mycosphaerella fijensis) en Granada mostró un grado cinco de afectación 34.5\%, mientras que en Fuente de Oro presentó un grado cuatro, 19.5\%, siendo superior el daño en comparación con los demás tratamientos en los cuales se utilizó Hipotensor y Ecotensor SYS con el fungicida, observando un grado de afectación de tres, puesto que no se superó el $15.6 \%$ de severidad de la enfermedad.

Las dosis de Ecotensor SYS, utilizadas para el control de la Sigatoka negra (Mycosphaerella fijensis), no generaron fitotoxicidad al cultivo del plátano. De acuerdo con los resultados en esta prueba de eficacia es de $1.0 \mathrm{ml}$ por litro de mezcla. Además, El Ecotensor SYS puede reemplazar al Hipotensor SYS que se utilizó en un tratamiento como referencia.

\section{REFERENCIAS BIBLIOGRÁFICAS}

1. Aguirre, SE; Piraneque, NV; Menjivar, JC. Relación entre las propiedades edafoclimáticas y la incidencia de Sigatoka negra (Mycosphaerella fijiensis Morelet) en la zona bananera del Magdalena-Colombia. Revista de Investigación Agraria y Ambiental, 3 (2): 13-23. 2012. Disponible En: https://dialnet.unirioja.es/servlet/articulo?codigo $=5344950$

2. Alarcón, J; Jiménez, Y. Manejo fitosanitario del cultivo del plátano (Musa spp): Medidas para la temporada invernal (en línea). ICA (Instituto Colombiana Agropecuario). Bogotá. 2012. Disponible En: https://www.ica.gov.co/getattachment/08fbb48d-a985-4f96-98890e66a461aa8b/-nbsp;Manejo-fitosanitario-del-cultivo-de-platano.aspx

3. Álvarez E, Pantoja A, Gañán L, Ceballos G. La Sigatoka negra en plátano y banano. Guía para el reconocimiento y manejo de la enfermedad, aplicado a la agricultura familiar. CIAT y FAO. 2013. Disponible En: http://www.fao.org/3/as089s/as089s.pdf

4. Aránzazu, F, Arcila M, Bolaños M, Castrillón C, Castellanos P, Pérez J, Rodríguez J, Valencia J. El cultivo de plátano: manual técnico. Corpoica, Manizales. 114 p. 2002. Disponible En: https://repository.agrosavia.co/handle/20.500.12324/17751

5. Arrospide, G. Criterios para el uso de aditivos y Coadyuvantes. Carrasco- Uruguay: Calister S.A. 2004. Disponible En: https://www.calister.com.uy/wpcontent/uploads/2016/06/1311182916Criterios para el uso de Aditivos y Coadyuv antes.pdf

6. Cuéllar A, Álvarez E, Castaño J. Evaluación de resistencia de genotipos de plátano y banano a la Sigatoka negra (Mycosphaerella fijiensis Morelet.) Rev. Fac. Nal. Agr. Medellín, 64 (1): 5853-5865. 2011. Disponible En: http://www.scielo.org.co/pdf/rfnam/v64n1/a11v64n01.pdf

7. Espinal C, Martinez H, Peña, Y. La cadena del banano en Colombia. Una mirada global de si estructura dinámica 1991-2005. Ministerio de Agricultura y Desarrollo Rural. Documento de trabajo N. 60. Bogotá. 2005. Disponible En: http://hdl.handle.net/11348/5874

8. Fernández V, Sotiropoulos T, Brown, P. Formulaciones y adyuvantes en: fertilización foliar, principios científicos y práctica de campo. Asociación Internacional de la Industria de Fertilizantes (IFA). Paris, Francia, p 37-48. 2015. Disponible En: 
https://www.researchgate.net/publication/283908842 Fertilizacion Foliar Principios Cientificos y Practicas de Campo/citation/download

9. González C, Arévalo E, Díaz L, Galindo J, Rosmira M, Guerrero M, Jimenez J, Manejo fitosanitario del cultivo de plátano (Musa sp). Medidas para temporada invernal. ICA. Bogotá - $\quad$ Colombia. $2012 . \quad$ Disponible En: https://www.ica.gov.co/getattachment/08fbb48d-a985-4f96-9889-0e66a461aa8b/nbsp;Manejo-fitosanitario-del-cultivo-de-platano.aspx

10. INTAGRI. Coadyuvantes para potencializar el rendimiento de plaguicidas. Serie Fitosanidad N. 94. Artículos Técnicos de INTAGRI. México. 8 p. 2017. Disponible En: https://www.intagri.com/articulos/fitosanidad/coadyuvantes-para-potencializar-elrendimiento-de-los-plaguicidas

11. Mejía J. Cultivo de plátano (Musa paradisiaca) Centro Nacional de Tecnología Agropecuaria y Forestal Enrique Álvarez Córdova. 2018. Disponible En: http://centa.gob.sv/docs/guias/frutales/Guia\%20Centa Platano\%202019.pdf

12. Orozco M, Orozco J, Pérez O, Manzo G, Farías J, Da Silva W. Prácticas culturales para el manejo de la Sigatoka negra en bananos y plátanos. Tropical Plant Pathology, 33 (3): 189-196, 2008. En: https://www.scielo.br/j/tpp/a/sfk79TX5GLKJHfYH6ymrVTB/?lang=es\&format=pdf

13. Orozco M, García K, Manzo G, Guzmán S, Martínez L, Beltran M, Garrido E, Torres J Y, Canche C. La Sigatoka negra y su manejo Integrado en banano. Primera Edición Publisher: INIFAP. Editor: Mario Orozco Santos. Consejo Estatal de Productores de Plátano del Estado de Colima A.C. 2013. Disponible En: https://www.researchgate.net/publication/256297564 La Sigatoka negra y su man ejo Integrado en banano

14. Puentes B. Coadyuvantes agrícolas, propiedades y uso en aspersiones de plaguicidas. ARROZ, $\quad p \quad 21-31.2011 . \quad$ Disponible http://www.fedearroz.com.co/revistanew/arroz495.pdf

15. Ramos P, Peñaranda M. Criterios de selección de coadyuvantes. Metflor-agro, 2018. Disponible En: https://www.metroflorcolombia.com/criterios-de-seleccion-decoadyuvantes/

16. Rivas, G., Rosales, F. (ed). Manejo convencional y alternativo de la Sigatoka negra, nematodos y otras plagas asociadas al cultivo de Musáceas en los trópicos. Actas del Taller "Manejo convencional y alternativo de la Sigatoka negra, nematodos y otras plagas asociadas al cultivo de Musáceas", celebrado en Guayaquil, Ecuador. 11-13 de Agosto. 2003. Disponible En: https://www.bioversityinternational.org/fileadmin/ migrated/uploads/tx news/Manejo c onvencional y alternativo de la Sigatoka negra nematodos y otras plagas asoc iadas al cultivo de Mus\%C3\%A1ceas en los tr\%C3\%B3picos 1242.pdf

17. Sazo L, Araya J, De la Cerda J. Efecto del coadyuvante siliconado e insecticidas en el control del chanchito blanco de la vid, Pseudococcus viburni (Hemiptera: Pseudococcidae). Cien. Inv. Agr. 35 (2): 215-222. 2008. Disponible En: https://scielo.conicyt.cl/pdf/ciagr/v35n2/art12.pdf

18. Stover, R. H. A proposed international scale for estimating intensity of banana leaf spot. Tropical Agriculture 48: 185-196. 1971. Disponible En: https://journals.sta.uwi.edu/ta/index.asp?action=viewlssue\&issueld=313 